\title{
Sensitivity enhancement of fiber Bragg gratings to transverse stress by using microstructural fibers
}

\author{
Charles Jewart, Kevin P. Chen, Ben McMillen, Michael M. Bails, and Steven P. Levitan \\ Department of Electrical and Computer Engineering, University of Pittsburgh, 3700 O'Hara Street, Pittsburgh, \\ Pennsylvania 15261 \\ John Canning \\ Optical Fibre Technology Centre, University of Sydney, 206 National Innovation Centre, Eveleigh 1430, Sydney, New \\ South Wales, Australia \\ Ilya V. Avdeev \\ ANSYS Inc., Canonsburg, Pennsylvania 15371 \\ Received April 21, 2006; revised April 26, 2006; accepted May 4, 2006; \\ posted May 15, 2006 (Doc. ID 67589); published July 10, 2006

\begin{abstract}
We present simulation and experimental results of fiber Bragg grating responses to transverse stress in microstructure fibers. The grating wavelength shifts and peak splits are studied as a function of external load and fiber orientation. Both simulation and measurement results indicate that the sensitivity of grating sensors to the transverse stress can be enhanced by a factor of eight in a two-hole fiber over that in a standard fiber. () 2006 Optical Society of America
\end{abstract} \\ OCIS codes: $060.2370,060.2280$.
}

Fiber Bragg gratings (FBGs) have been widely used as strain gauges. Although most of the strain measurements performed by FBG sensors have been along the longitudinal axis of the fiber, there is increased interest in measuring strain transverse to the fiber. ${ }^{1,2}$ The transverse stress distribution has been previously measured by a number of fiber sensor elements, including FBGs in highly birefringent fibers. ${ }^{1}$ The birefringence induced by the external load leads to both shifts and splitting of FBG peaks of two orthogonal polarization modes, which can be used to gauge the magnitude and the orientation of the transverse strain field. To enhance the sensitivity, FBGs in multicore fibers were recently used to measure transverse stresses. ${ }^{2}$ By comparing FBG peak splitting and shift in fiber cores located in different locations of the fiber, both the magnitude and the orientation of the transverse stress were measured with a single fiber.

In this Letter we present a simple alternative approach to directly improve the sensitivity of fiber sensors to transverse stress by using microstructure fibers. FBGs in air-structured photonic crystal fibers were recently used to separate temperature- and longitudinal-strain-induced FBG shifts. ${ }^{3}$ We demonstrate here that by proper fiber design the sensitivity of a FBG sensor to transverse stress can be significantly enhanced by the introduction of air holes in the fiber cladding. The design of the microstructure fiber is similar to that used in civil engineering but on a micrometer scale. Instead of placing a fiber core near the edge of the fiber, the core is fabricated in the fiber center for convenience of splicing. A number of air holes are strategically placed in the fiber cladding to alter the stress distribution, which can be used to either focus the stress into the fiber core, or deflect stress from the core. The introduction of air holes also breaks the symmetry of the fiber structures, since they are placed asymmetrically. Thus fiber sensors become sensitive to both the magnitude and the orientation of the external force. This idea is first illustrated in this Letter by using a finite element analysis (FEA) simulation and subsequently confirmed experimentally by using a fiber with a two-airhole design.

In a solid fiber, when a transverse load is applied vertically to the fiber, the maximum stress takes place in the vicinity of the contact point. In the area of the fiber center, the stress is dispersed across the entire fiber because of the increased area of the reaction plane in the fiber center. This is confirmed by a FEA using ANSYS 8.0, shown in Fig. 1(a). Therefore the sensitivity of fiber Bragg gratings in the fiber core to transverse stress is reduced dramatically compared with a core at the edge of the fiber. However, since the stress is measured by pressure per unit area, the stress induced in the fiber core can be focused by reducing the reaction area in the fiber center. This can be achieved by introducing air holes in the fiber. A two-hole fiber is used in this Letter to illustrate the concept. Two-hole fibers have been previously used for poling and hydrostatic pressure measurement. ${ }^{4,5}$ Figure $1(\mathrm{~b})$ shows the stress distribution along the direction of external load ( $y$ axis, dominate stress component) in a two-hole fiber. Two air holes reduce the reaction area in the fiber center and effectively focus the stress into the fiber core located between the two air holes. Comparing fibers of the same diameter $(220 \mu \mathrm{m})$, the introduction of two air holes with a diameter of $90 \mu \mathrm{m}$ increases compression stresses [negative stress in Figs. 1(a) and 1(b)] by 6 times that at the center of the solid fiber. 


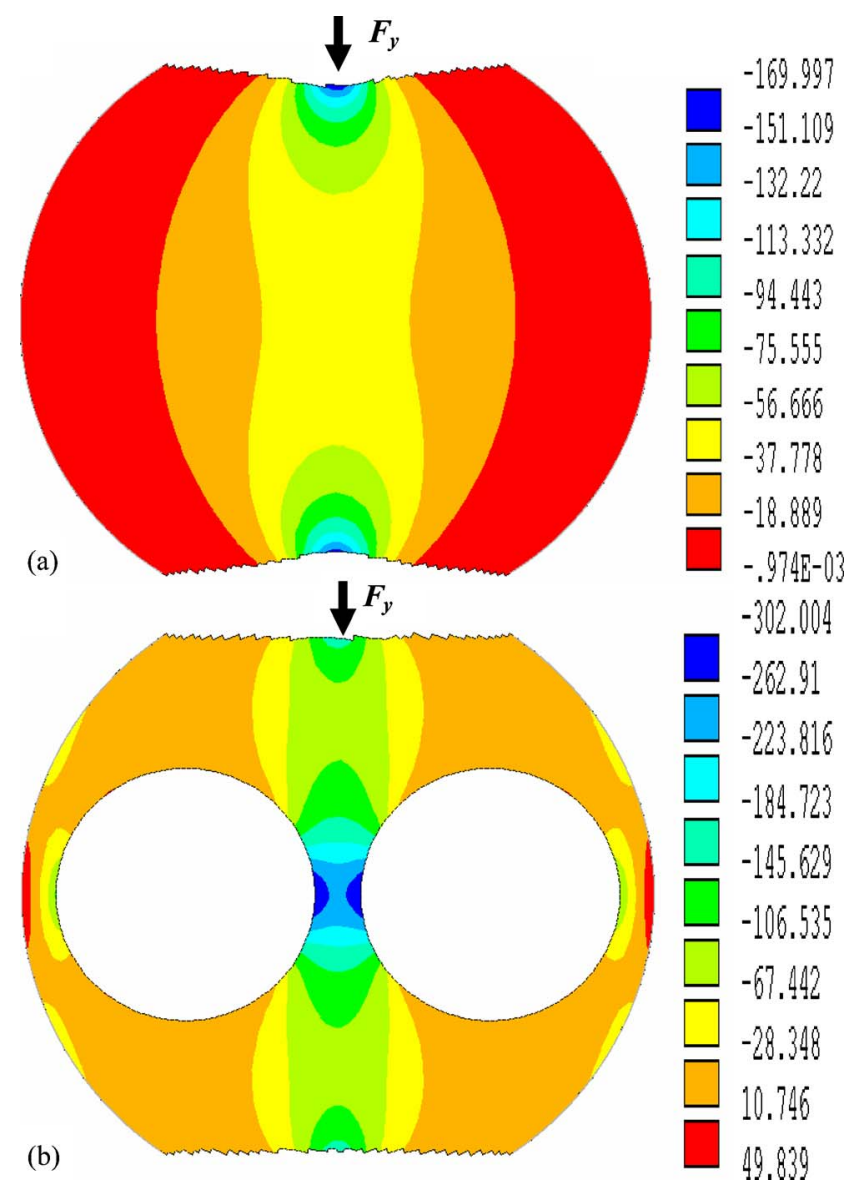

Fig. 1. (Color online) Simulated vertical stress distribution $\sigma_{y}$ of (a) a $220-\mu \mathrm{m}$ diameter solid fiber and (b) a $220 \mu \mathrm{m}$ diameter two-hole fiber under an external load of $F_{y}=50 \mathrm{~N} / \mathrm{cm}$. The units for the scale bar are megapascals. The top and bottom contact points of the external force are truncated to show detailed stress distribution in the fiber center.

The introduction of air holes also breaks the symmetry of the fiber; thus the response of the fiber to external load becomes orientation sensitive. This can be illustrated by the stress distribution along the section defined by the centers of the two air holes and the center of the fiber, shown in Fig. 2. When two air holes are oriented perpendicular to the external load, compression stresses (negative stress) are produced along both the $x$ and $y$ directions. This is in contrast to a tensile stress (positive stress) produced by the load when two air holes are parallel to the force. The dominant stress in the fiber core for both fiber orientations is approximately 11 times that in the other axis, which will lead to a significant birefringence in the fiber core and FBG peak splitting. ${ }^{6}$ Figure 2 also indicates that the maximum stress is produced on the edge of the air holes owing to a large deformation around the air-hole area. Therefore, to maximize the sensitivity of FBG sensors, the fiber core should be placed right beside the air holes.

Based on these simulation results, a twin-hole fiber (provided by the Optical Fiber Technology Center) with a diameter of $220 \mu \mathrm{m}$ was chosen for experimental validation as shown in Fig. 3. Two air holes were drilled into a fiber preform by using an ultra- sonic driller, which produced two $90 \mu \mathrm{m}$ diameter holes in the fiber cladding after the fiber was drawn. An elliptical fiber core with a long axis of $9.7 \mu \mathrm{m}$ and a short axis of $7.5 \mu \mathrm{m}$ was fabricated $1 \mu \mathrm{m}$ from the edge of the air hole to maximize the sensitivity to transverse stress. The outer edge of the air holes was $10 \mu \mathrm{m}$ from the fiber edge. To verify the simulation results, $1 \mathrm{~cm}$ FBGs were written in the twin-hole fibers by a $248 \mathrm{~nm} \mathrm{KrF}$ excimer laser by a standard phase mask technique. The configuration of the loading test experiment was similar to that used in Ref. 2 and is briefly described here. First, the twin-hole fiber was mounted on a rotational stage to adjust the orientation of two air holes to the external load. The orientation of the air holes was monitored by a CCD microscope mounted at the end of the fiber. The test fiber and a dummy fiber were mounted between two flat, polished metal plates. The transverse stress was carefully applied by a spring-loading apparatus and was monitored by a load cell underneath the bottom metal plate. The length of the fiber under pressure was $80 \mathrm{~mm}$. A broadband amplified stimulated emission source, an optical spectrum analyzer, and a single-mode fiber coupler were used to monitor the FBG under test.

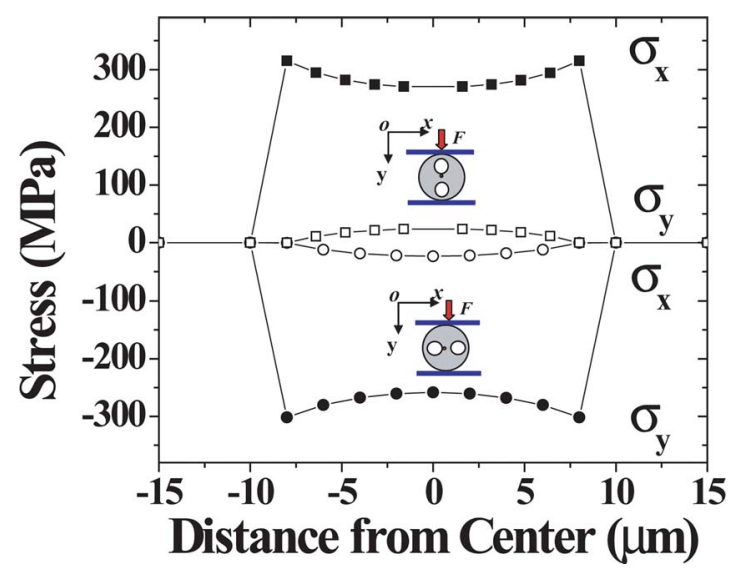

Fig. 2. (Color online) Vertical and horizontal stress distributions of a fiber section defined by the centers of the fiber and centers of two air holes under a load of $50 \mathrm{~N} / \mathrm{cm}$.

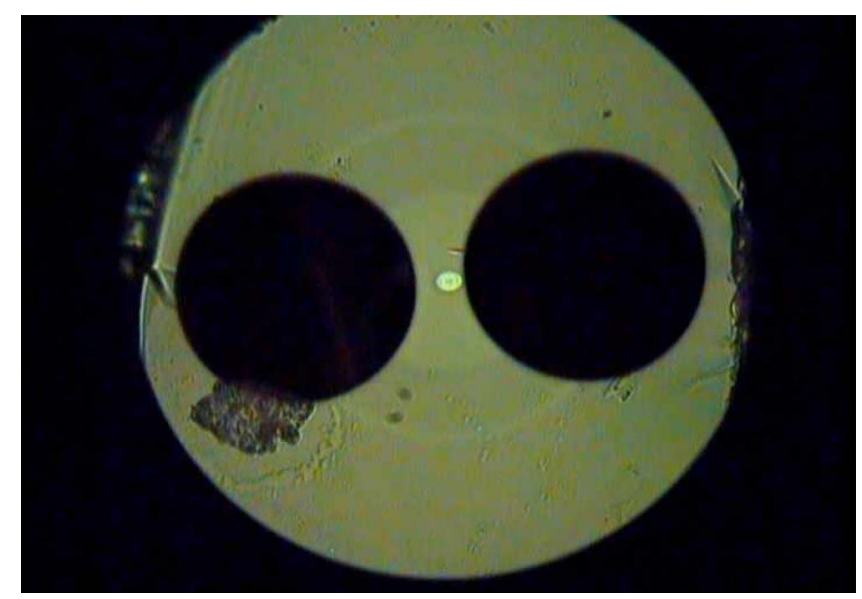

Fig. 3. (Color online) Optical microscope picture of a cross section of a two-hole fiber. The bright spot between the two air holes is the fiber core. 


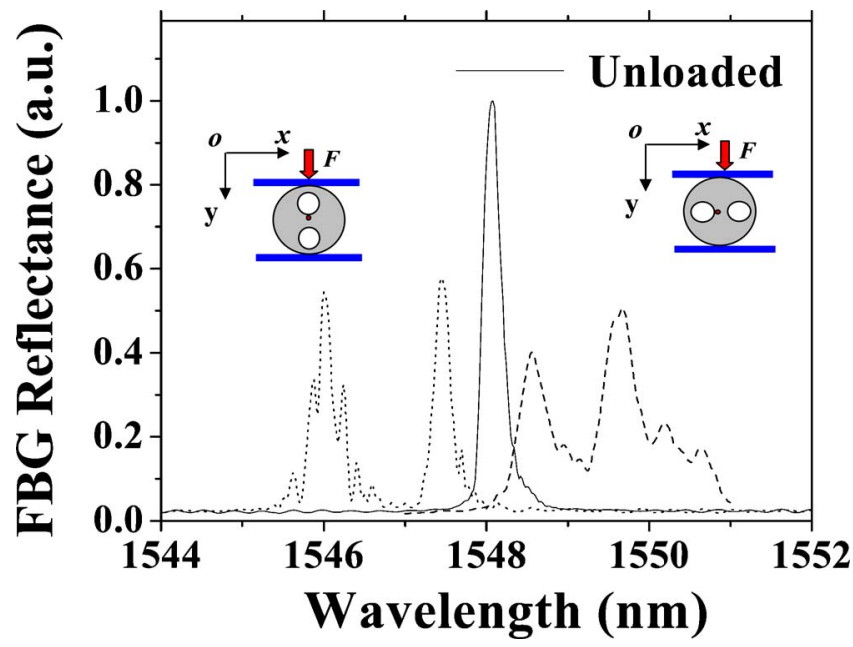

Fig. 4. (Color online) Reflection spectra of a FBG in a twohole fiber when two air holes were oriented perpendicular and parallel to an external load of $80 \mathrm{~N} / \mathrm{cm}$.

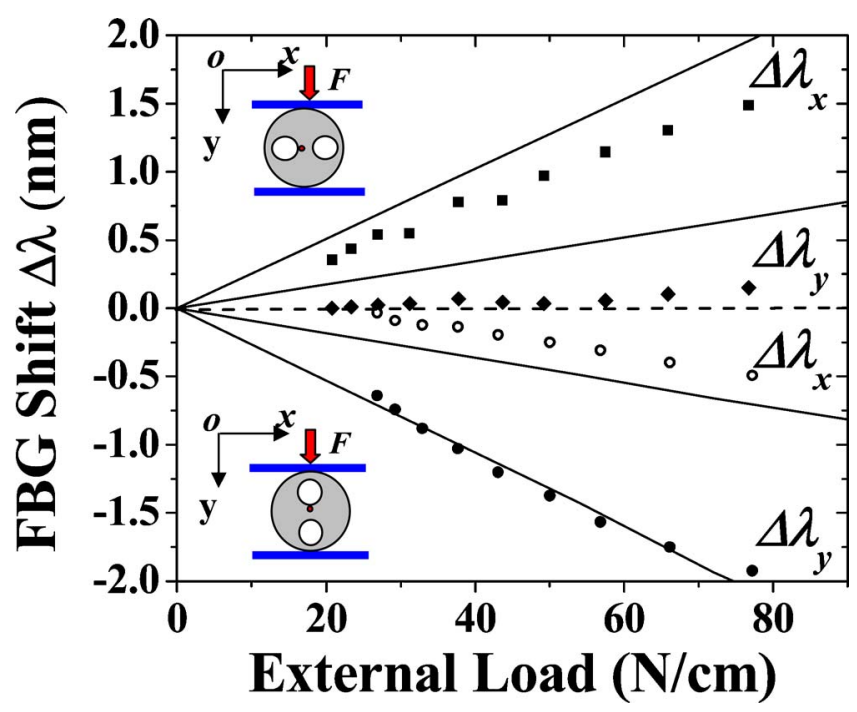

Fig. 5. (Color online) FBG peak shifts and splits in a twohole fiber as a function of an external load for both perpendicular and parallel hole orientations. Solid lines are FEA simulation results.

The reflected spectra of the FBG under an external load are shown in Fig. 4. When the two air holes were oriented perpendicular to the load of $80 \mathrm{~N} / \mathrm{cm}$, the FBG peaks shifted to shorter wavelengths and produced a splitting of $1.7 \mathrm{~nm}$, which corresponds to an induced birefringence $\Delta n \sim 1.7 \times 10^{-3}$. In contrast, when the two air holes were parallel to the external load of $80 \mathrm{~N} / \mathrm{cm}$, a redshift of the FBG peak and a splitting of $1.2 \mathrm{~nm}\left(\Delta n \sim 1.2 \times 10^{-3}\right)$ was observed. The simulation results are qualitatively consistent with these measurements. As a comparison, an
$80 \mathrm{~N} / \mathrm{cm}$ load produces only a $0.3 \mathrm{~nm}$ redshift and a $0.05 \mathrm{~nm}$ blueshift for $x$ and $y$ polarizations in a FBG written in a $125 \mu \mathrm{m}$ diameter standard fiber.

To better explain the behavior of the two-hole FBG under transverse loading, the simulated results are compared with the experimental results in Fig. 5. The model predicts a linear shift of FBG peaks, represented by solid lines in Fig. 5. The simulation yields a shift in the FBG peak at a rate of $26 \mathrm{pm} /(\mathrm{N} / \mathrm{cm})$ for the dominant stress axis and $17.6 \mathrm{pm} /(\mathrm{N} / \mathrm{cm})$ for the peak splitting, respectively, which is 8 times and 4 times that for a $125 \mu \mathrm{m}$ diameter solid fiber.

The loading experiments were performed with an external load up to $80 \mathrm{~N} / \mathrm{cm}$. Well-defined FBG peak splitting due to the birefringence induced by the external loads was clearly identified for a force exceeding $20 \mathrm{~N} / \mathrm{cm}$. Figure 5 also shows a discrepancy between the experimental results and the simulation. The simulation predicts the same magnitude of FBG peak shifts for both horizontal and vertical hole orientations but in opposite directions. However, FBG peak shifts for the $y$ polarization are four times less than the theoretical prediction for the horizontal hole orientation. Further studies are currently under way to investigate this discrepancy.

In summary, this paper illustrates a concept to enhance the sensitivity of fiber sensors to transverse loads by using microstructure fiber design. This work suggests that highly sensitive strain, bending, and acoustic fiber sensors can be developed by using microstructure fibers.

The authors thank Simon Fleming at the Optical Fiber Technology Centre, University of Sydney for valuable discussions and for supplying two-hole fiber samples. Helpful suggestions from Manuel SilvaLopez and William N. MacPherson at Heriot-Watt University (Edinburgh, UK) are acknowledged for the loading experiment setup. K. P. Chen's e-mail address is kchen@engr.pitt.edu.

\section{References}

1. C. M. Lawrence, D. V. Nelson, E. Udd, and T. Bennett, Exp. Mech. 39, 202 (1999).

2. M. Silva-Lopez, C. Li, W. N. MacPherson, A. J. Moore, J. S. Barton, J. D. C. Jones, D. Zhao, L. Zhang, and I. Bennion, Opt. Lett. 29, 2225 (2004).

3. C. Martelli, J. Canning, N. Groothoff, and K. Lyytikainen, Opt. Lett. 30, 1785 (2005).

4. T. Fujiwara, D. Wong, and S. Fleming, IEEE Photon. Technol. Lett. 7, 1177 (1995).

5. E. Chmielewska, W. Urbańczyk, and W. J. Bock, Appl. Opt. 42, 6284 (2002).

6. R. Gafsi and M. A. El-Sherif, Opt. Fiber Technol. 6, 299 (2000). 